\title{
The structure of reflection as the basis of the procedural organization of consciousness
}

\author{
Anatoly V. Karpov \\ P. G. Demidov Yaroslavl State University, Yaroslavl, Russia
}

Corresponding author. E-mail: anvikar56@yandex.ru

\begin{abstract}
The relationship of consciousness to the reflexive processes is one of the fundamental problems in psychology. To date, however, many important aspects of this problem remain poorly understood. This article attempts a theoretical study of this problem. The subject of research is the interconnection between consciousness and reflexive processes and also the structure of these processes. This study presents theoretical and empirical materials revealing the interrelatedness of these two fundamental subjects of psychological research. In connection with this issue, I introduce a new methodology for psychological research, which I refer to as the metasystem approach. This approach is grounded in the claim that reflection is the basic procedural means of consciousness and is based on a heterarchical principle. This article proposes a new structural, polyprocess method for reveaing the psychological nature of reflection and the contents of reflexive processes. I propose a new explanation for the basic properties of the psyche that underlie consciousness: self-sensitiveness (sensitivity to oneself). I theoretically prove the following proposition: reflection has a fundamental heterogeneity because its processes are localized on completely different hierarchical cognitive levels of personality. In addition, I show that in the structural organization of reflection as an integral process in relation to other ("secondary," metacognitive) processes, another basic principle is involved - the principle of hierarchy. The property of reflexivity (and the process of reflection as a procedural manifestation of this property) should be understood as a species in relation to the more common attribute inherent in the psyche: self-sensitiveness. Elementary manifestations of this property have been observed for the simplest sensory processes.
\end{abstract}

Keywords: consciousness, reflection, metasystem approach, heterarchy, structure, metacognitive processes, levels, self-sensitiveness

\section{Introduction}

In the psychological literature, one common position is that consciousness, as both an object of scientific research and, correspondingly, a gnoseological display, possesses a number of specific, even unique, characteristics. The most obvious among 
them is an intensive combination of complexity and significance in the development of psychological knowledge in general. The gnoseological condition, according to which the complexity of a problem and its significance are in direct relation to and derivative of each other, is, as F. M. Dostoevsky (1872/1972) described it, "the last" frontier of psychological exploration. Gnoseology is also the most significant theoretical issue in psychology, as the constructive solution of almost all other major problems in psychology is directly connected with it and, moreover, depends on it. Consciousness has a completely objective quality because consciousness itself has properties of "distributiveness" and "omnipresence": it is an organizing and, as a rule, primary starting point in almost all basic formations, processes, and structures of the psyche. Similarly to the way consciousness pervades all behavior and underlies its organization, the underlying qualities of consciousness also permeate psychological theory and exploration.

Another specific feature of consciousness (both as an object and as a problem) is the interchangeable notions of the cognizing and the cognizable. In "the face" of consciousness, these elements are not only largely identical but also equally powerful and of the same essence. This relationship, in turn, gives rise to the fundamental problem of the possibility (or impossibility) of "cognizing the cognizable by means of this very cognizable," which is a kind of epistemological analogue to the range of tasks that provide the reason for K. Gödel's formulation of his famous "incompleteness theorem" (Gödel, 1951/1995).

Finally one of the main peculiarities of consciousness is that it is the highest form (and level) of psychic integration. All basic components of mental processes and the main modes of their existence are presented in indissoluble unity and organic wholeness. Consciousness simultaneously acts as a unique characteristic of human beings, as a special property, as a specific state, and as a system of the processes that deliver it. However, it is an organic whole. In other words, the integrity and complexity of consciousness create the inherent difficulty of researching it, a difficulty that is clearly manifested in the peculiarities of the modern state of the problem. Consciousness (as a subject of psychological research) is most clearly and fully presented in its final, effective manifestations - that is, as property, state, and ability. Thus, the study of these manifestations acts as the basic, most traditional, and most widely presented direction in the development of the general problem of consciousness. The concept of consciousness is, above all, well known: for example, Searle's "anti-dichotomous concept" (1992); Chalmers's "non-reductionist" concept (1996); Rosenfeld's "self-reference" concept (in Wellman, Crooss, \& Watson, 2001); Velmans's theory of reflexive consciousness (2000); Ey's integrative concept (1983); Baars's cognitive concept (1988); Priest's synthetic concept (2000).

However, there is every reason to believe that the bases of all these results are actually procedural means and mechanisms employed to ensure the very existence of consciousness. They, however, are implicit and therefore difficult to study (but at the same time are important). They should provide a transition from a productive aspect of the study of consciousness to the procedure for studying those mental processes that underpin it. In fact, such a transition is equivalent to the transition from research as a phenomenon to a study of its nature. In this regard, such research is indicative of the general logic of the development of powerful trends in modern cognitive psychology, which constitute metacognitivism. The fact is that 
the main subjects of this research are just those mental (metacognitive) processes that are largely the procedural basis of consciousness. It is quite natural that a number of concepts currently in metacognitivism need to be applied to the problem of consciousness in general in order to develop summative, holistic representations concerning its organization and functioning. Particular examples are Tobias and Everson's hierarchical model of metacognition (Tobias, Everson, \& Laitusis, 1999); Metcalfe's synthetic concept of metacognition (Metcalfe \& Shimamura, 1994); Ferrari's hierarchical model of metacognitive processes (in Wellman et al., 2001); Sternberg's ideas about metacomponents in the overall structure of intelligence (1985); Dörner's theory of “cognitive metaoperators" (1977); Wellman's theory about the "soul of man" (Wellman et al., 2001); Brown's theory about the structure of metathinking (1987); Jarman's concept of "synthetic metaprocesses" (Jarman, Vavrik, \& Walton, 1995); Karaliotas's "reflexive concepts” of metacognition (1998); Blaky and Spens's theory of the "metaarchitectonics of consciousness" (in Wellman et al., 2001). However, the two fundamental areas of psychological knowledge interdisciplinary modern cogitology (the "science of consciousness") and metacognitivism - have developed largely independently. In this regard, there is reason to believe that reaching a conceptual synthesis of these two areas can significantly contribute to the resolution of the problem of consciousness. On this basis, the main purpose of this article is to consider the procedural plan of the mind that is based on the views that have been developed regarding the metacognitive system and, in addition, the reflexive processes.

Thus, a priority and objectively critical issue in the study of consciousness is the disclosure and explanation of the relationships between reflection and consciousness, as well as their psychological structure.

\section{Results}

The uniqueness of the problem of consciousness is determined by the fact that consciousness as a summarizing phenomenon and reflection as its leading procedural means are inherent only in human beings and set them apart from all other living beings. Consciousness is the basis of successful human existence. However, another aspect of the consciousness problem is the fact is that almost all other problems in psychology can and should be understood as products of the science of psychology itself - both its theoretical and clinical development and its consequences ${ }^{1}$. However, the concept of psychology in the broad and original sense of the word, as "knowledge of the soul," is objectively possible only on the basis of the property of reflection and the ability to be conscious. And in this respect we can say that all psychology is the consequence of this property, which appears wherever and whenever possible. Reflection allows us to differentiate between the act of "cognizing" and the object that is "cognizable." Although psychology as knowledge of the soul is only one form of knowledge, all knowledge is possible only in its reflective context. Therefore, one of the most burning questions in psychology - What is knowledge? - is identical in many respects to the question What is consciousness? 
Consciousness denotes the form and the conditions that allow for the existence of any knowledge, including scientific and, especially, psychological knowledge.

The above-mentioned characteristics do not exhaust the specification and uniqueness of consciousness because this very uniqueness is fundamentally plural. Consciousness is exhaustively examined in the psychological literature. However, among the main peculiarities of the problem of consciousness, at least one has not been given due attention and is not subject to independent methodological reflection, although it is this very peculiarity that will shed further light on our understanding of the nature of consciousness.

The inherent and unprecedented complexity of consciousness precludes generalized methodological approaches to studying it. In fact, this complexity interferes with the heuristics designed to allow methodological approaches. To characterize this relationship, it seems appropriate to refer to a well-known expression by Plekhanov, who formulated it with respect to freedom of will: "It, like a mysterious Sphinx, gazes at any newly proposed philosophical doctrine and says: «solve me, or I will devour you»" (1956, p. 211).

An essential point of the above position is that it stimulates the need to develop new methodological approaches, new paradigms of psychological research. In some cases this perspective even considers hypertrophied manifestations because it is in the development of the problem of consciousness that rather unusual and extravagant, although valuable, psychological approaches are sometimes created. Attempts have been made to develop explanatory approaches to the nature of consciousness as, for instance, being like gravity or being a completely separate entity on the same order with matter and energy. However, rather than changing the general meaning of the marked peculiarity, these "epistemological aberrations" only emphasize its importance and indicate that the main criterion for new methodological approaches should be their constructiveness and continuity with respect to positive findings that have been established through the use of other theoretical and experimental approaches.

Because of the complex nature of consciousness - and the fact that it has as yet eluded accurate understanding by psychology experts - it is important to develop new epistemological and methodological tools for continued research on it. One potential tool, which I have proposed (Karpov, 2004), is a metasystem approach; it can be summarized as follows. According to this method, to achieve full disclosure of particular objects of psychological research and of the psyche in general, it is necessary to focus on the contents of the system itself; these contents will be thus represented in the system's essential features and therefore will constitute a specific part of the system. The most obvious and demonstrative illustration of this position is the basic principles of the organization of the psyche as a whole. Indeed, external, objective reality (as the metasystem with which the psyche initially interacts) takes on a kind of "double" existence in the form of subjective or "reflected" (to use the traditional terminology) reality. This subjective reality can take different forms designated and interpreted variously according to its mechanisms, structures, and processes; however, its existence is undeniable and indisputable. Therefore, the attributive nature of the psyche and its uniquely reflective nature create the dual objective/subjective reality described above. Moreover, the more precisely subjective reality corresponds to objective reality, the greater the number of prerequisites that 
are provided for solving general adaptation tasks. Therefore, it can be stated that the metasystem with which the psyche initially interacts and that is objectively included and "externally positioned" in the psyche appears as part of the structure and content of the psyche itself. In other words, it becomes transposed onto the psyche, although in the highly specific form of subjective reality.

Thus, the very essence of the psyche is that, in its own content, the metasystem, which is initially externally positioned to the psyche, appears to be presented within the psyche and gains the double existence of objective/subjective reality. The level at which this interaction of the metasystem and the psyche is accomplished is transposed onto the content of the psyche. As a result, the psyche acquires a qualitatively new status, becoming a system on the "built-in" metasystem level. I emphasize here once more that I am referring only to a specific form of the existence of this objective reality, not to its ontological representation in the psyche.

In addition, as shown by my and I. M. Skityaeva's previous studies (Karpov \& Skityaeva, 2005), not only the psyche as a whole but also its other core components are organized on the basis of the above-mentioned principle and are therefore qualitatively specific, unique systems. These systems have also been identified by us as being on the built-in metasystem level. One of the specific and unique features of these components in relation to the consciousness problem is the following peculiarity. On the one hand, a system functions according to its own autochthonous patterns; otherwise, it would be impossible to speak of any formation, process, structure, and so on as of a qualitatively specific, independent, ontologically presented entity. On the other hand, however, a system also includes (if only functionally) the metasystem but does not cease to be a part of this metasystem. Thus, there is a unique and somewhat unusual, paradoxical, but also objective auto-interaction in which the system is able to interact with itself without violating its own objective regularities. In this context, it is possible to assume that the metasystem is generic in relation to the self-sensitiveness of the psyche as a species property; however, this property of self-sensitiveness is most relevant to the psychological nature and specific character of consciousness. Therefore, auto-interaction can and should be included as a basic explanatory means for the study of consciousness.

Undoubtedly, we face here the most crucial issue for developing an understanding of the consciousness problem as a whole: the disclosure of mechanisms underlying the phenomenon of auto-interaction. In my opinion, a solution to this problem is impossible without further deepening and elaborating in detail ideas about the nature, specific character, and content of the reflective processes. The category of consciousness and the concept of reflection, although not coinciding in general and although also being in a fairly complex and ambiguous relationship, are inextricably attributively connected to each other. Through the reflective regulation of activities, behavior, and communication, all known (and unknown) mental processes can be defined holistically as an organized structure. And this integrity (and, hence, completeness) attaches a conscious character to reflective regulation and creates a subjective "feeling of complete control" that is usually denoted by concepts of the "conscious character of behavior," the "report of one's actions," and so forth.

However, at present the concept of reflection is still rather vague and insufficiently differentiated, as it has no clearly explicit structural characteristics. At 
this point difficulties occur in the theoretical interpretation of the unprecedentedly high heterogeneity of the content and procedural forms of reflection. In addition, a large number of its kinds, types, forms, means, and modes are empirically fixed. Furthermore, this heterogeneity poses a fundamental question: Is reflection a unitary process, or is the term reflection cumulative, and, in fact, is some specific system of reflective processes forming the overall organization of the mental processes?

In an attempt to answer this question, I consider it necessary to formulate the following basic positions. When analyzing reflective phenomena and processes, it is accepted that they are heterogeneous: nevertheless, this "diversity" does not qualify for independent and special analysis, as there is no question about its causes and meaning. However, the manifestations and phenomenology of this heterogeneity are indisputable and invite questions about the existence of some significant underlying elements on which it is based. The range of reflective processes and phenomena within consciousness is unprecedented - from elementary vague "selfawareness" to highly deployed, refined, and sophisticated forms of self-cognition. And this extremely high degree of heterogeneity within reflective processes and phenomena has served and continues to serve as one of the main challenges to creating an adequate conceptualization of the reflection process.

The key to solving the problem of the procedural status of reflection exists precisely within this extreme heterogeneity of reflection. In fact, it seems rather strange and even paradoxical that, in the diverse procedural manifestations of reflection, a fundamental regularity has not been recognized and recorded. In both psychological terminology and the vernacular, a number of concepts and expressions have been developed for identifying procedural manifestations of reflection: self-sensation, self-perception, auto-presentations (or auto-concepts), "self-directed" attention, "memory of memory" (metamemory), and "thinking of thinking (metathinking). Significantly, according to modern psychological interpretation, all these processes belong to the category of metacognitive processes and constitute as a whole their most important component (Couchman, Coutinho, Bera, \& Smith, 2010; Kelly \& Metcalfe, 2011; Kieran \& Fox, 2014; Kluwe, 1982; Kolb, 1995; Yzerbyt, Lories, \& Dardenne, 1998). In these concepts, the different levels of reflection correlate with basic cognitive processes. From these claims, a significant conclusion follows: reflection as a process and as one of the macrolevels in the overall organization of the psyche is built according to the level principle. Thus, reflection reproduces basic levels of the cognitive hierarchy, and each sublevel of reflection fully, accurately, directly, and naturally correlates to one or another basic level of the cognitive hierarchy. The cognitive hierarchy, in the face of reflection, turns into the inner contents of the psyche but performs the same functions that it would in relation to the external environment. In this context, it is possible to speak of two forms of the cognitive hierarchy: externally oriented and internally oriented. The economy and practicality of the psyche's organization lie in the fact that a single system manifests in these substantially different forms.

From the above standpoint, it is possible not only to describe reflection processes more comprehensively than previously but also to clarify and expand on the principles of the multileveled organization of mental processes. When these principles are applied in psychological research, a direct correlation and even mu- 
tual conditionality between this organization and the hierarchical character of its construction have become somewhat axiomatic. In simpler words, if there are levels, they are synthesized in a structure that, in turn, has to be integrated into a hierarchy. At the same time, the psychological study of reflection shows that it is still a special case of correlated, multileveled organizational principles and hierarchical principles. They involve each other when a system directly implements controlling, regulatory functions. In addition, they can be realized through reflection itself, taken in its regulatory mode (currently indicated by the terms cognitive monitoring and metacognitive involvement in the activity); see the review in Karpov (2011).

However, in its attributively original form and qualitative certainty, the essence of reflection is somewhat different. On the one hand, the nature of the reflective process seems to "interrupt" a behavioral continuum, suspending its direct implementation. Thus, reflection acts independently in that it has its own cognitive mode. However, as a fundamentally unique task of self-presentation, reflection cannot and, apparently, should not be built hierarchically, although it is organized according to the leveled-structure principle. To clarify, among all the processes included in reflection (ranging from self-sensation to metathinking), in principle it is impossible to consider any of these as the "most important" oricess and therefore as the process "on the top of the hierarchy." For reflection as a procedural means of consciousness (and for consciousness as a whole), the psyche's self-sensation is not less than, and may be more significant than, for example, the ability "to think of yourself" (that is, metathinking). The essence of reflection lies in the fact that it achieves an exhaustive representation of the inner world in all its manifestations, including those that are procedural. This representation implies a reliance on all cognitive processes, taken in their "secondary" forms as metaprocesses. Thus, the above representations regarding the structural organization of reflective processes in particular and metacognitive processes in general contribute to the attainment of one of the most important objectives of modern metacognitivism: the disclosure of the patterns of the structural organization of this class of processes as "secondary" in that they form a qualitatively different level in relation to the "primary" mental processes (Kieran \& Fox, 2014; Kuhn, 2009; Metcalfe \& Shimamura, 1994). Their structure takes the shape of a heterarchical organization and represents procedural contents, namely, the phenomenon of consciousness in general.

Based on the above description, the structure-level organization of mental processes is maintained, but the hierarchical organization is not. Thus it can be concluded that the leveled structure is not always associated with a hierarchy, and, instead, a hierarchy is only one manifestation of the leveled structure. In the system of reflective processes, structural levels are of the same order, but complete selfawareness implies a reliance on all these levels working simultaneously and equally. I emphasize that a heterarchical organization applies only to the system of cognitive processes as a whole, not to reflection specifically. In my opinion, the psychological nature of reflection is such that not only are thoughts expressed, but it is impossible not to express them. From this standpoint, reflection as a procedural aspect of consciousness is a heterarchy of cognitive metaprocesses. Its essence, as is known, consists in the presence of two (or more) parity "control centers" at the same time, and it is, according to existing theoretical concepts, more sophisticated and powerful 
than the hierarchical principle of organization. Furthermore, reflection is the most important integrator of mental processes. However, the essence of this integration lies in the fact that, on the one hand, it is unfolding on the basis of the heterarchy principle, and, on the other hand, the main mental processes, or "objects" of this integration, have a sort of double existence when modified as metacognitive, or secondary, processes.

\section{Conclusion}

The methodological significance of the above conclusion reaches far beyond the bounds of reflection. First, it becomes obvious that the integration of any system (in this case cognitive processes) is not always or "automatically" deployed on the basis of a subordinate principle. In fact, the notions of integration and hierarchy are not at all mutually conditional. Integration as a phenomenon and the integrative mechanisms that support it can be qualitative rather than hierarchical. This integrative process, apparently, is even more complicated than the principle of hierarchy and needs further study to be fully understood.

Second, within psychology, the fundamental problem of self-organization and self-organizing systems cannot be solved hierarchically. As I have shown (Karpov, 2011), the self-organization of complex and hypercomplex systems cannot objectively be implemented only on the basis of a hierarchy; there must be parity of at least several if not all of its major subsystems in addition to a large number of "controlling, organizing centers" in the system.

Thus, the hierarchical organization of the reflective processes includes a set of heterogeneous procedural means formed from the evolution of a fundamental property of the psyche: self-sensitiveness, or sensitivity to itself and its content. This self-sensitiveness, in turn, is engendered by a combination of cognitive metaprocesses. From these positions, two important psychological characteristics of reflection are revealed clearly:

1. Reflection as understood in its broadest sense has a fundamental heterogeneity because its processes are presented on different planes of the metacognitive hierarchy. A traditional understanding of reflection sees only its most deployed form as the basis on which metathinking lies.

2. The property of reflectivity (including reflection as a procedural manifestation of this property) should be understood as being related to a general and attributively inherent property of the psyche: self-sensitiveness, the elementary manifestations of which are observed in the simplest sensory processes. With this specific feature, any cognitive process acts not only in its original form or primary functional purpose as a means of processing information but also as an object that has an active transformational impact on other mental processes, including itself. In this case, each process of reflection as a whole appears not only as an active operator but also as a relatively passive operand, and the reversibility of these modes underlies the entire reflective process. Thus, one means of explaining auto-interaction is as a mechanism attributively inherent in cognitive processes that can be defined as operand-operatory reversibility. 
The ideas presented in this article allow one to form conclusions about a more general plan related to identifying the most promising directions for the development of the problem of consciousness. These ideas provide a constructive (and even necessary) conceptual synthesis of two fundamental areas of psychological knowledge: interdisciplinary areas of modern cogitology (the science of consciousness) and metacognitivism. Such a synthesis is central to solving the key problems that are formulated in cogitology (in particular, in the concepts of Searle, Chalmers, Rosenfeld, Clark, Wellman, Ey, Baars, Priest) and in metacognitivism (in the works of Tobias, Everson, Ferrari, Sternberg, Dörner, Metcalfe, Wellman, Brown, Blaky, Spens). The basis of all phenomenological results - that is, the effective manifestations and properties of consciousness - are actually procedural means and mechanisms employed to ensure its existence and functioning. So there must be disclosure of a transition from a productive aspect of the study of consciousness to the procedure for studying the mental processes that underpin it. In fact, such a transition is equivalent to the transition from research as a phenomenon to a study of its nature. Implementation of this approach, as presented above, has enabled the government to disclose and describe the system of the processes that make up the actual content of the procedural components of consciousness. At the same time, this approach allows us to give a new interpretation of a common set of reflective processes, showing them to be a logically organized and structured procedural means of consciousness.

\section{Acknowledgements}

The research is performed with the financial support of the Russian President's grant for the leading Russian scientific schools, \# HШ-2165.2014.6.

\section{References}

Baars, B. J. (1988). A cognitive theory of consciousness. Cambridge, U.K.: Cambridge University Press.

Brown, A. L. (1987). Metacognition, executive control, self-regulation, and other more mysterious mechanisms. In F. E. Weinert \& R. H. Kluwe (Eds.), Metacognition, motivation, and understanding (pp. 65-116). Hillsdale, NJ: Erlbaum.

Chalmers, D. J. (1996). The conscious mind: In search of a fundamental theory. Oxford, U.K., New York: Oxford University Press.

Couchman, J., Coutinho, M., Bera, M., \& Smith, J. (2010). Beyond stimulus cues and reinforcement signals: A new approach to animal metacognition . Journal of Comparative Psychology, 124(4): 356-368.

Dörner, D. (1977). Problem-solving as processing of information. Stuttgart: Springer-Verlag.

Dostoyevsky, F. M. (1972). Demons. In Collected works Fiction literature. Vol. 8. (Original work published 1872).

Ey, H. (1983). Consciousness. Paris: Gallimard, 1983.

Gödel, K. (1995). Some basic theorems on the foundations of mathematics and their implications. In S. Feferman (Ed.), Kurt Gödel: Collected works: Unpublished essays and lectures (Vol. 3, pp. 304-323). New York: Oxford University Press. (Original work published 1951)

Jarman, R. F., Vavrik, J., \& Walton, P. D. (1995). Metacognitive and frontal lobe processes: At the interface of cognitive psychology and neurophysiology. Genetic, Social, and General Psychology Monographs, 121, 153-210. 
Karaliotas, Y. (1998). Electronic surveys as metacognitive tool. Honolulu: University of Hawaii. Karpov, A. V. (2004). The metasystem organization of level structures of the psyche. Moscow: IP RAN.

Karpov, A. V. (2011). The psychology of consciousness. The metasystem approach. Moscow: RAO. Karpov, A. V., \& Skityaeva, I. M. (2005). The psychology of the metacognitive processes of personality. Moscow: IP RAN.

Kelly, K., \& Metcalfe, J. (2011). Metacognition of emotional face recognition. Emotion, 11, 8696. doi: 10.1037/a0023746

Kieran, C. R., \& Fox, K. (2014, February). Metacognitive facilitation of spontaneous thought processes: When metacognition helps the wandering mind find its way. Cognitive Neuroscience of Metacognition, 293-319.

Kluwe, R. (1982). Cognitive knowledge and executive control: Metacognition. In D. R. Griffin (Ed.), Animal mind-human mind (pp. 201-204). New York: Springer.

Kolb, S. K. (1995). Metacognition metaphors. New York: Oxford University Press.

Kuhn, D. (2009). The importance of learning about knowing: Creating a foundation for development of intellectual values. Child Development Perspectives, 3(2), 112-117. doi: 10.1111/ j.1750-8606.2009.00089.x

Metcalfe, J., \& Shimamura, A. P. (Eds.). (1994). Metacognition: Knowing about knowing. Cambridge, MA: MIT Press.

Plekhanov, G. V. (1956). To the question of the role of personality in history. In Selected philosophical works (Vol. 2, p. 408). Moscow: Science.

Priest, S. (2000). Theories of consciousness. Moscow: IdeaPress.

Searle, J. R. (1992). The rediscovery of the mind. Cambridge, MA: MIT Press.

Sternberg, R. J. (1985). Beyond IQ. Cambridge, U.K.: CUP.

Tobias, S., Everson, H. T., \& Laitusis, V. (1999). Towards a performance based measure of metacognitive knowledge monitoring: Relationships with self-reports and behavior ratings. Paper presented at the annual meeting of the American Educational Research Association, Montreal.

Velmans, M. (2000). Understanding consciousness. London: Routledge.

Wellman, H. M., Crooss, D., \& Watson, J. (2001). Meta-analysis of theory-of-mind development: The truth about false beliefs. Child Development, 72 (3), 655-684. doi: 10.1111/14678624.00304

Yzerbyt, V. Y., Lories, G., \& Dardenne, B. (Eds.). (1998). Metacognition: Cognitive and social dimensions. London: Sage.

Original manuscript received February 03, 2015

Revised manuscript accepted July 27, 2015

First published online September 30, 2015 